\title{
Modelling of Direct Ultraviolet Photoionization and Charge Recombination of Aerosol Nanoparticles in Continuous Flow
}

\author{
R. Nishida, ${ }^{1,}$ a) A. Boies, ${ }^{1}$ and S. Hochgreb ${ }^{1}$ \\ Department of Engineering, University of Cambridge, Cambridge CB2 1PZ, UK
}

(Dated: 28 November 2016)

Ultrafine aerosol particles are electrically charged in a range of devices to enable their detection, capture and control. Direct ultraviolet (UV) photoionization enables increased charging of some nanoparticle materials over alternative charging mechanisms such as diffusion charging, particularly in size ranges below $50 \mathrm{~nm}$ diameter. The aim of this work is to provide modelling and simulation of ion and particle charge and discharge processes, transport and collection in a continuous flow. A non-dimensional analysis indicates regimes under which the photocharging process is dominated by diffusion, electric field transport, convection, photoionization, or recombination. The computational fluid dynamics (CFD) model developed in this work is the first to include equations capturing UV photoionization and detailed ion and particle recombination theory. The validity of assumptions made for diffusional wall losses and external electric field action are evaluated by comparison with OD Numerical and 3D CFD models. Regimes are identified to distinguish the level of detail required for aerosol transport and charging models.

\section{INTRODUCTION}

The study of aerosol particle photoionization has developed over the past several decades after it was discovered that ultraviolet light could provide high efficiency electric charging of aerosol particles ${ }^{1}$. Direct ultraviolet (UV) photoionization enables increased charging of some nanoparticle materials over alternative charging mechanisms such as diffusion charging ${ }^{2-4}$. By directly charging particles using sufficiently high energy photons, higher particle charge states can be reached which are not limited by electrostatic repulsion with like-charged ions ${ }^{2,4}$. The higher states of particle charging and collision free charging mechanism provide opportunities for improvements in sensing ${ }^{5-7}$, capture ${ }^{8}$ and control of aerosol particles.

After the initial development of particle photocharging theory ${ }^{9-12}$, aerosol photocharging studies to date have largely focused on the experimental development of polyaromatic hydrocarbon (PAH) sensors for industrial hygiene or combustion control ${ }^{5,6,13-17}$ or to study and enhance charging of nanoparticles below $20 \mathrm{~nm}$ diameter $2,4,8,18-21$. Modelling of the photoionization process has not seen significant improvement since the application of the Fowler-Nordheim equation in a $0 \mathrm{D}$ case with recombination by Maisels et al. in $2003^{22-24}$.

In this work we model the behaviour of UV particle charging and subsequent charge transport and collection in a continuous flow, as a means to evaluate photoionization theory and provide tools for quantitative evaluation of ultrafine particle charge states. This work aims to quantify the effect of a low-strength electric field which is commonly employed to remove ions and thereby reduce particle-ion recombination after the photoionization process. Non-dimensional analysis is used to indicate

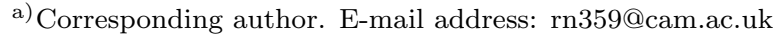

regimes under which the photocharging process is dominated by diffusion, electric field transport, convection, photoionization, or recombination. In particular, the ratio of characteristic irradiation to recombination time defines the regime when charge saturation is reached, which is a function of particle size, concentration, material, and radiation wavelength and intensity. The physical processes of photoionization and recombination of ions and particles are modelled in 3D computational fluid dynamics (CFD) for the first time. The detailed terms obtained in the 3D model in the charge balance equation are analysed to understand the range of validity of assumptions made for diffusional wall losses and external electric field in $0 \mathrm{D}$ analytical models. Recommendations are made regarding the level of detail required for the prediction of aerosol charging and capture methods over a range of conditions used in experiments or the predictive design of photocharging-based devices.

\section{ANALYTICAL AND NUMERICAL MODELLING}

\section{A. Particle Charging Theory and Governing Equations}

Particles are charged directly by absorbing incoming UV photons and emitting electrons, which differs from diffusive charging that relies on the process of ion-toparticle collision or recombination. When photons of sufficient energy are absorbed, the particles emit electrons, which in turn collide with the surrounding air and form gaseous ions. The remaining particle develops a positively charged electrostatic field which increases the photon energy required to release additional electrons. Photoelectric emissions occur when the energy of an irradiating photon is higher than the work function of the particle surface. The original Fowler-Nordheim equation for flat surfaces is extended to solve for the probability of for photocharging spherical particles from charge level $q$ to $q+1$, called a combination coefficient ${ }^{9,22}, \alpha^{q \rightarrow q+1}$, 


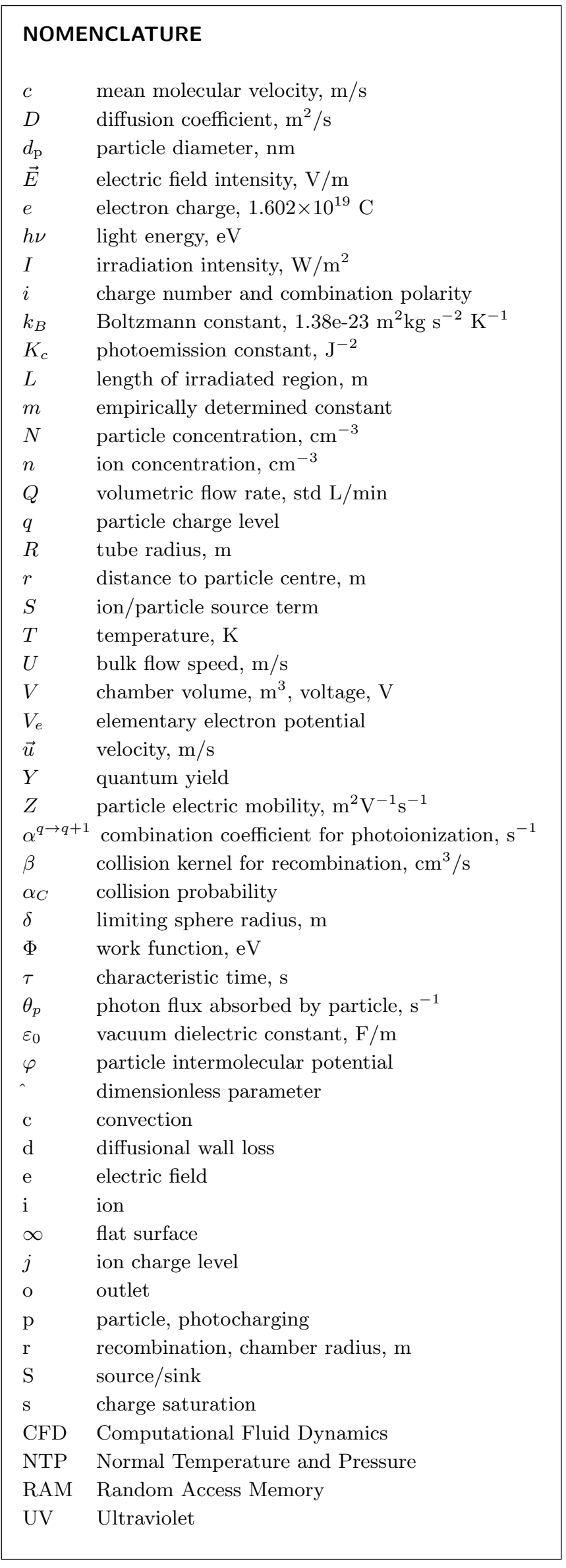

$$
\alpha^{q \rightarrow q+1}=\underbrace{K_{c}\left(h \nu-\Phi^{q \rightarrow q+1}\right)^{m}}_{\text {quantum yield }} \underbrace{\frac{I \pi d_{\mathrm{p}}^{2}}{h \nu}}_{\begin{array}{c}
\text { photon } \\
\text { flux } \\
\left(\mathrm{s}^{-1}\right)
\end{array}} .
$$

The probability $\alpha^{q \rightarrow q+1}$ is proportional to the product of the photon flux, given by the ratio of the intensity of UV irradiation, $I$, to specific photon energy, $h \nu$, and the photoelectrically active area of the particle, $\pi d_{\mathrm{p}}^{2} / 4$. The specific photon energy, $h \nu$, must be greater than the particle surface work function, $\Phi$, to result in a positive emission probability, and the proportionality function increases with the excess specific energy raised to a power $m^{7}$. The value of the exponent $m$ has been determined as around 2 for metals and a few non-metals ${ }^{12,18,25}$, while one study has shown that $m=3$ for diesel particles ${ }^{26}$. The proportionality constant $K_{c}$ is a material dependent and empirically determined. Equation 1 is valid within the Fowler-Nordheim regime $(h \nu-\Phi \lesssim 1.5 \mathrm{eV})$. Outside the Fowler-Nordheim regime, increasing photon energy decreases photoelectric yield, a phenomenon which has not yet been explained by physical principles ${ }^{27}$.

Once an electron is emitted, the work function, $\Phi$, increases according to:

$$
\begin{aligned}
\Phi^{q \rightarrow q+1} & =\Phi_{\infty}+\frac{2 V_{e}}{d_{\mathrm{p}}}\left(q+1-\frac{5}{8}\right) \\
V_{e} & =\frac{e^{2}}{4 \pi \varepsilon_{0}}
\end{aligned}
$$

where $\Phi_{\infty}$ is the work function of a flat surface of the same material and $V_{e}$ is the elementary electron potential. The $q+1$ term represents the Coulomb force between a released electron and the remaining charged particle which must be overcome by the photon energy to emit the electron. After each electron is released, the Coulomb force increases and opposes further release. The $\frac{5}{8}$ term represents the difference in image force between a flat surface and a spherical particle, to account for the fact that the spherical particle has less attraction to the released electron ${ }^{11}$.

As long as the incident photon energy, $h \nu$, is greater than the work function, the particle continues to charge to a maximum level of charges per particle, $q_{\max }$, given by:

$$
q_{\max }=\frac{2 d_{\mathrm{p}}}{V_{e}}\left(h \nu-\Phi_{\infty}\right)-\frac{3}{8} .
$$

The $\frac{3}{8}$ term represents the image charge effect on $q_{\max }$ and may be neglected for $q_{\max } \gg 1$. The real maximum charging is limited by recombination effects, deglomeration or in extreme cases, Coulomb explosion ${ }^{2}$. 


\section{Ion-particle Recombination}

The emitted electrons from photoionization attach to gas molecules (e.g. oxygen) or impurities within $\sim 10^{-5} \mathrm{~s}$, creating gaseous ions, referred to henceforth as ions ${ }^{1,28}$. The electric mobility of the resulting ions is on the order of 100 times greater than the remaining charged particles in the size range of interest (10-300 nm), depending on particle size and charge level. The gaseous ions may diffuse back to and transfer charge to the charged particles, thereby neutralizing them, as illustrated in Fig. 1. If ions are removed due to diffusional wall losses or by capture in a low-strength electric field, ions are less likely recombine with the charged particles, thereby increasing the particle charge level. In an irradiated region, both photoionization and recombination take place, whereas in a UV irradiation free region, only recombination is possible.

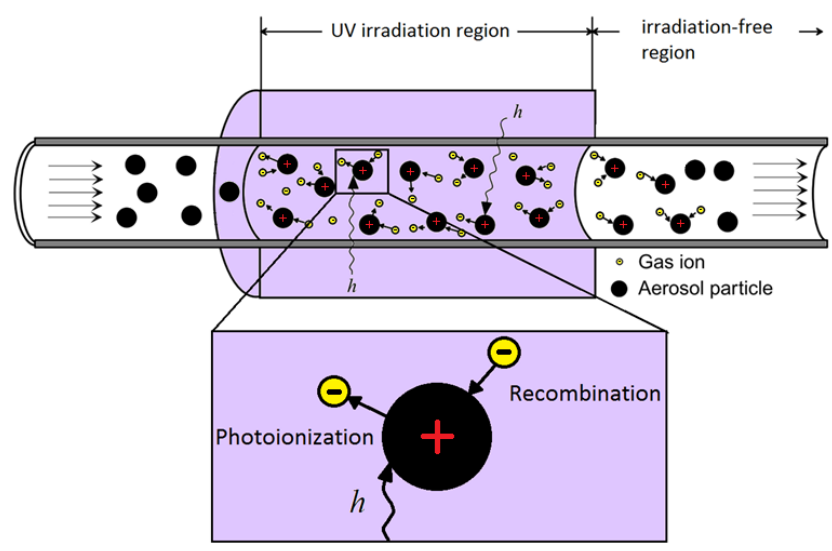

FIG. 1. Photoionization and recombination of particles entrained in flow.

Recombination proceeds according to the same collision processes between ions and particles associated with diffusion charging or neutralization. The collision kernel, $\beta$, calculated using the method of Hoppel and Frick $^{29,30}$, accounts for diffusive and electrostatic forces to quantify the rate of recombination. Efforts have been made to develop improved collision kernel expressions to supplant or extend the range of validity of the Hoppel and Frick method ${ }^{31,32}$, sometimes including ion mobility distributions ${ }^{33}$. These methods may be readily employed in this model if required by the application. For details of the collision kernel calculation used in this work, see Reischl et al. ${ }^{34}$ which contains a useful summary of the Hoppel and Frick ${ }^{29}$ method which is summarized as

$$
\begin{aligned}
\beta & =\frac{\pi \delta^{2} c \alpha_{C}(q)}{\exp \left(\frac{\varphi(i(q), \delta)}{k_{B} T}\right)+\frac{\delta c \alpha_{C}(q)}{4 D_{i}} \int_{0}^{1} \exp \left(\frac{\varphi(i(q), \delta / x)}{k_{B} T}\right) d x} \\
\varphi(i, r) & =V_{e}\left(\frac{i}{r}-K \frac{\left(d_{p} / 2\right)^{3}}{2 r^{2}\left(r^{2}-\left(d_{p} / 2\right)^{2}\right)}\right)
\end{aligned}
$$

where the limiting sphere radius, $\delta$, is a function of the mean free path of the ions ${ }^{34}, c$ is the mean molecular velocity, and $\alpha_{C}$ is the ion-particle collision probability which depends on charge and distance. The potential $\varphi$ is a function of the material and particle diameter, where $K=1$ for a perfectly conducting particle, $r$ is the distance from the centre of the particle and $i$ is the number and polarity of particle charges such that $i$ is positive if the ion and particle are of the same polarity. A collision of an ion with a particle assumes that the ion transfers its charge to the particle so that the population balance for either leads to a source term in the concentration equations.

\section{Governing Equations}

Photoionization changes ion and particle charge states, acting as a source term for ions and charged particles, which can be recombined or transported by convection, diffusion or electric forces. The steady state conservation equations for the number of particles of a given charge $q$, and ions of charge $j$ in a differential control volume is given as:

$$
\begin{aligned}
\nabla \cdot\left(\vec{u} N_{q}\right)=\nabla \cdot\left(D_{p} \nabla N_{q}\right)+\nabla & \cdot\left(Z_{q} \vec{E} N_{q}\right) \\
& +S_{q, \alpha}+S_{q, \beta} \\
\nabla \cdot\left(\vec{u} n_{j}\right)=\nabla \cdot\left(D_{i} \nabla n_{j}\right)+\nabla & \cdot\left(Z_{j} \vec{E} n_{j}\right) \\
& +S_{j, \alpha}+S_{j, \beta}
\end{aligned}
$$

where $N_{q}$ is the concentration of particles of a given size and material at $q$ charge level, $n_{j}$ is the concentration of ions with $j$ charge level, $\vec{u}$ is the velocity of the surrounding fluid, $D$ is the ion or particle or ion diffusivity, and $S$ are the source/sink terms for photocharging, $\alpha$, and recombination, $\beta$. The contribution to the particle balance due to the applied electric field transport is represented by particle electric mobility, $Z$, subject to the electric field, $\vec{E}$ (Eq. 5a).

The source/sink terms $S$ represent the rate of increase or decrease in the number concentration of the particles or ions owing to either photoionization or recombination via particle to ion collisions. For example, if a negative ion, $n_{-1}$, attaches to a particle with a single positive charge, $N_{+1}$, the particle reduces to a neutral charge level, $N_{0}$, and the ion returns to a neutral gas molecule. Here we assume that the concentrations of neutral gas molecules greatly exceed those of ions or charged particles, so that their concentrations remain unchanged. Only singly, negative ionized gas molecules of monodis$d x$ perse mobility represented by the number concentration $n_{-1}$ are considered: the photoionisation process causes the emission of (negative) electrons, which are assumed to immediately collide with a gaseous molecule, generating a negative ion. Assuming a monodisperse aerosol of 
diameter $d_{p}$ and a single material type, the source terms in Eq. 5 are given as follows:

$$
\begin{gathered}
S_{q, \alpha}=\alpha^{q-1 \rightarrow q} N_{q-1}-\alpha^{q \rightarrow q+1} N_{q} \\
S_{q, \beta}=\beta^{q+1 \rightarrow q} N_{q+1} n_{-1}-\beta^{q \rightarrow q-1} N_{q} n_{-1} \\
S_{-1, \alpha}=\sum_{q=q_{\min }}^{q_{\max }} \alpha^{q \rightarrow q+1} N_{q} \\
S_{-1, \beta}=-\sum_{q=q_{\min }}^{q_{\max }} \beta^{q \rightarrow q-1} N_{q} n_{-1}
\end{gathered}
$$

where $\alpha^{q \rightarrow q+1}$ is the photoionization-related combination coefficient for photoionization calculated from Eq. 1, and $\beta^{q \rightarrow q-1}$, is the so-called collision kernel for the recombination of an ion with a particle of charge $q$. The collision kernel is a function of particle diffusivity and interparticle potential, and is calculated using Eqn 4. Equations 5 and 6 are solved for all charge levels, $q$, between a minimum and maximum, where the minimum charge is set sufficiently negative to include the minimum expected charge level and neutral particles. The full governing equations may be solved using 3D numerical methods, as described in section II C, but a simplified model suggested by Maisels et al. ${ }^{22}$ is considered below as a reference case, as it can be solved analytically.

\section{B. Simplified Analytical Model}

Part of the difficulty in solving the conservation equations comes from the non-linearity in the source terms. In the present paper, we take a limit case considered by Maisels et al. by assuming that the charges per particle are large and positive ${ }^{22}$. In that case, it is possible to neglect the image terms, and the combination coefficients can be expressed as:

$$
\begin{gathered}
\alpha^{q \rightarrow q+1}=\frac{K_{c} I}{h \nu} \frac{\pi d_{\mathrm{p}}^{2}}{4}\left(h \nu-\Phi_{\infty}-\frac{2 q V_{e}}{d_{\mathrm{p}}}\right)^{m} \\
\beta^{q \rightarrow q-1}=4 \pi D_{i} \frac{V_{e}}{k_{\mathrm{B}} T} q
\end{gathered}
$$

Further, the source terms of Eqns. 6 can be approximated as moments in the charge distribution, in the limit of large number of charges per particle, so that the sums are replaced by a function of mean charge per particle $\bar{q}$, and total number of particles $N$, that is, $\bar{S}_{\alpha}=$ $\sum_{q} \alpha^{q \rightarrow q+1} N_{q}=\bar{\alpha} N$, and $\bar{S}_{\beta}=-\sum_{q} \beta^{q \rightarrow q-1} N_{q} n_{-1}=$ $-\bar{\beta} N n_{-1}$. An analytical solution can be obtained for the mean charge $\bar{q} N=\sum_{q} q N_{q}=n_{-1}$ for steady plug flow conditions with a constant total number concentration of particles $N$, where $\vec{u}=U \vec{e}_{x}$ :

$$
U \frac{\mathrm{d} n_{-1}}{\mathrm{~d} z}=\bar{S}_{\alpha}+\bar{S}_{\beta}
$$

Maisels et al. considered the limits to Eq. 9 for (a) zero recombination losses, so that $\bar{S}_{\beta}=0$, and (b) steady state conditions after a sufficient convection length (or time) under irradiation, where $\frac{\mathrm{d} n_{-1}}{\mathrm{~d} z}=0$. For the zero recombination case we have:

$$
\begin{aligned}
\bar{q}_{z \rightarrow 0} & =\frac{z}{U \tau_{\alpha}}=\frac{t}{\tau_{\alpha}} \\
\tau_{\alpha} & =\left[\frac{K_{c} I}{h \nu} \frac{\pi d_{\mathrm{p}}^{2}}{4}\left(h \nu-\Phi_{\infty}\right)^{m}\right]^{-1}
\end{aligned}
$$

where the residence time is defined as $t=z / U$. The irradiation time, $\tau_{\alpha}=\left(\bar{S}_{\alpha, z \rightarrow 0}\right)^{-1}$, is the inverse of the characteristic rate of photon generation, which is the product of the the photon rate absorbed by the particles, $\frac{1}{\tau_{\nu}}=\frac{I \pi d_{\mathrm{p}}^{2}}{4 h \nu}$, and the quantum yield at zero mean charge, $Y=K_{c}\left(h \nu-\Phi_{\infty}\right)^{m}$, where $\tau_{\alpha}=\tau_{\nu} / Y$. After a sufficient residence time under irradiation, $\tau_{s}$, a steady state level of charges per particle, $\bar{q}_{s}$, is found when either the extent of recombination equals that of photocharging, as obtained from setting the left hand side of Eq. 9 to zero, so that $\bar{S}_{\alpha}+\bar{S}_{\alpha}=0$, or when the Coulomb limit is reached. Using $m=2$ and expanding the brackets in Eq. 7, the mean steady state charge can be obtained as:

$$
\begin{array}{r}
\bar{q}_{t \rightarrow \infty}=\bar{q}_{s}=q_{\max }\left(1+\sqrt{\hat{A}_{v}}\right)^{-1} \\
\hat{A}_{v}=\frac{4 h \nu D_{i} N}{K_{c} I V_{e} k_{\mathrm{B}} T}
\end{array}
$$

where $\hat{A}_{v}$ represents the ratio of recombination over photoionization and recombination must be considered when $\hat{A}_{v} \gg 0$. The full equation for the mean charge has been derived by Maisels et al. ${ }^{22}$ as:

$$
\bar{q}=q_{\max } \frac{\tanh \left(\frac{\sqrt{\hat{A}_{v}}}{q_{\max } \tau_{\alpha}} t\right)}{\tanh \left(\frac{\sqrt{\hat{A}_{v}}}{q_{\max } \tau_{\alpha}} t\right)+\sqrt{\hat{A}_{v}}} .
$$

The square root term arises due to the factor $m=2$, which leads to an expansion of the quadratic term in the solution of the differential equation.

\section{Characteristic Saturation Time}

By equating Eqs. 10 and 11 with each other, we define a characteristic charge saturation time, $\tau_{\mathrm{s}}$, after which the photoionization rate matches the recombination rate and maximum charging occurs: 
TABLE I. Characteristic times

\begin{tabular}{|l|c|c|}
\hline & $\begin{array}{c}\text { Characteristic } \\
\text { times } \\
\text { (ions) }\end{array}$ & $\begin{array}{c}\text { Characteristic } \\
\text { times } \\
\text { (particles) }\end{array}$ \\
\hline Photocharging & $\tau_{\mathrm{p}, \mathrm{i}}=\frac{n_{-1} / N}{\bar{\alpha}}$ & \\
Recombination & $\tau_{\mathrm{r}, \mathrm{i}}=\frac{1}{\bar{\beta} N}$ & \\
Diffusion & $\tau_{\mathrm{d}, \mathrm{i}}=\frac{R^{2}}{4 D_{i}}$ & $\tau_{\mathrm{d}, \mathrm{p}}=\frac{R^{2}}{4 D_{p}}$ \\
Electric field & $\tau_{\mathrm{e}, \mathrm{i}}=\frac{R}{Z_{i} E}$ & $\tau_{\mathrm{e}, \mathrm{p}}=\frac{R}{Z_{p} E}$ \\
Convection & $\tau_{\mathrm{c}, \mathrm{i}}=\frac{L}{U}$ & $\tau_{\mathrm{c}, \mathrm{p}}=\frac{L}{U}$ \\
\hline
\end{tabular}

$$
\tau_{\mathrm{s}}=\frac{q_{\max } \tau_{\alpha}}{\left(1+\sqrt{\hat{A}_{v}}\right)} .
$$

The characteristic time, $\tau_{\mathrm{s}}$, therefore represents a minimum residence time to reach maximum particle charging. In a steady plug flow situation with volumetric flow rate across an area, $Q=U A$, this implies a minimum volume $V_{\min }=Q \tau_{s}$ to reach maximum particle charging. The dimensionless average charge per particle, $\bar{q}$, of Eq. 12 relative to the steady state level, $\overline{q_{s}}$, of Eq. 11 is shown as a function of the relative charging time, $t / \tau_{s}$, in Fig. 2, along with the limits from Eqs. 10 and 11. The dimensionless average charge level during the transition to steady state does not differ significantly for concentrations $10^{4}-10^{7} \mathrm{~cm}^{-3}$, when recombination is significant.

The mean charge is shown as a function of dimensional time and particle concentration in Fig. 3 for typical atmospheric particle concentrations. The average charge per particle decreases with increasing concentration due to the increased likelihood of recombination. The dashed lines in Figs. 2 and 3 are from Eqns. 10 and 11.

\section{Characteristic Times and Dimensionless Parameters}

In the present paper, we consider the effects not only of convection, charging and recombination, but also electric field transport and wall loss via diffusion. Therefore, it is useful to consider the characteristic times associated with the effect of each of the charging and transport mechanisms to identify which mechanisms are fast or slow for any given set of parameters. Characteristic times are defined for convection, $\tau_{\mathrm{c}}$, photocharging, $\tau_{\mathrm{p}}$, recombination, $\tau_{\mathrm{r}}$, wall loss, $\tau_{\mathrm{w}}$, and electric field transport, $\tau_{e}$, and are summarized in Table I. For example, the ratio of characteristic times for ion wall loss due to electric field

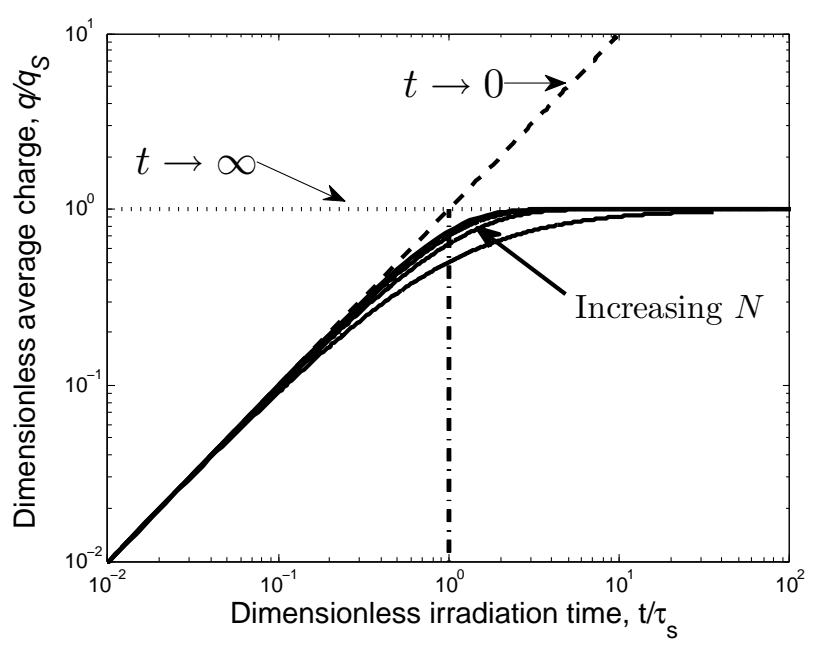

FIG. 2. Dimensionless average charge per particle, $\bar{q} / \bar{q}_{s}$, as a function of dimensionless time, $\tau_{c} / \tau_{s}$ for a range of concentrations.

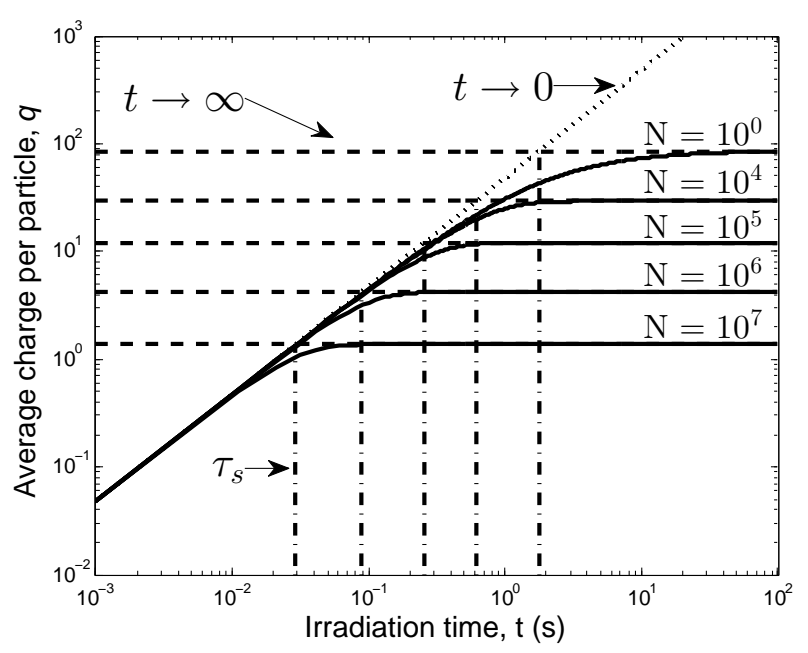

FIG. 3. Average charge per particle, $\bar{q}$, as a function of time for a range of concentrations using Eqs. 10 and 11. Characteristic time to charge saturation, $\tau_{s}$, is indicated by the vertical lines. The curved lines are generated from Eq. 12. Concentrations $N$ given in $\left[\mathrm{cm}^{-3}\right]$.

relative to the diffusional wall loss is:

$$
\hat{\tau}_{\mathrm{ed}, \mathrm{i}}=\frac{\tau_{\mathrm{d}, \mathrm{i}}}{\tau_{\mathrm{e}, \mathrm{i}}}=\frac{Z_{\mathrm{i}} E R}{4 D_{\mathrm{i}}} .
$$

For $\hat{\tau}_{\mathrm{ed}, \mathrm{i}} \ll 1$, ion capture at the walls due to electric field transport is slow relative to diffusional ion loss. For $\hat{\tau}_{\text {ed,i }} \gg 1$, electric field transport is fastest and dominant. Similar relations can be used to determine the relative effect of diffusional loss of ions and particles, for example, crucial in determining operating conditions of a given device.

The total charge flow can be estimated by ratios of the different characteristic times at the limits of, for example, full charging, where $\tau_{c}>\tau_{s}$. The number rates 
and electric currents are shown in Table II. For example, $i_{\mathrm{o}, \mathrm{p}}=Q N \bar{q} e$ gives the analytical limit estimate of the electric current available due to the flux of particles through the outlet, useful for a particle measurement device, while $i_{\mathrm{e}, \mathrm{i}}=Q \bar{q} N e \frac{\tau_{\mathrm{c}, \mathrm{i}}}{\tau_{\mathrm{e}, \mathrm{i}}}$ gives the corresponding magnitude of current due to the flux of trapped ions in an electric field during the photoionization process. The effect of electric field strength on both currents $i_{\mathrm{o}, \mathrm{p}}$ and $i_{\mathrm{e}, \mathrm{i}}$ are discussed in Section III using 0D numerical and 3D CFD models.

The dimensionless parameters give insight into the relative effects of particle and ion charging and transport mechanisms. They allow a simple estimation of particle and ion flux and electrical current due to the relative effects of convection, diffusional wall loss, and capture in an electric field. However, these estimates do not directly give the exact solutions for a given flow situation.

\section{Effect of Ion Loss on Particle Charge State}

Ions are less likely to recombine with particles if they are removed due to diffusional wall losses or electric field capture. Removing ions can significantly enhance particle charging. The following describes a simplified analytical method for calculating diffusional wall loss or electric field capture of ions to determine if ion loss has a significant effect on particle charge state.

Equations 5a and 5b may be rearranged assuming no particle wall losses and plug flow conditions, where $\vec{u}=$ $U \vec{e}_{x}$ :

$$
U \frac{\mathrm{d}}{\mathrm{d} z}\left(\bar{q} N-n_{-1}\right)=+\frac{4 D_{i}}{R^{2}} n_{-1}+\frac{Z_{j} E}{R} n_{-1} .
$$

Integration of Eq. 15 followed by linearization due to the small terms yields $\bar{q} N=n_{-1}\left(1+\hat{B}_{v}+\hat{C}_{v}\right)$, where the terms $\hat{B}_{v}$ and $\hat{C}_{v}$ are given as

$$
\begin{aligned}
& \hat{B}_{v}=\frac{\tau_{c}}{\tau_{\mathrm{d}, \mathrm{i}}}=\frac{4 D_{i}}{R^{2}} \tau_{c} \\
& \hat{C}_{v}=\frac{\tau_{c}}{\tau_{\mathrm{e}, \mathrm{i}}}=\frac{Z_{-1} E}{R} \tau_{c}
\end{aligned}
$$

where $\tau_{\mathrm{d}, \mathrm{i}}$ and $\tau_{\mathrm{e}, \mathrm{i}}$ are the characteristic times for diffusional wall loss and electric field loss, respectively. Parameters $\hat{B}_{v}$ and $\hat{C}_{v}$ represent the fraction of ions lost due to diffusion and electric field transport over time, respectively, valid for Pouiseuille flow in a cylindrical geometry. The diffusion and electric field loss terms are assumed to be small, so that $n_{-1}$ is taken as identical to that of the steady state solution $n_{-1,0}=\bar{q}_{0} N$ obtained in Eq. 11. The corrected mean particle charge is obtained for $\tau_{c}>\tau_{s}$, assuming $\tau_{s}$ is small:

$$
\bar{q}=q_{\max }\left(1+\sqrt{\frac{\hat{A}_{v}}{1+\hat{B}_{v}+\hat{C}_{v}}}\right)^{-1} .
$$

The assumption of small ion losses and no particle losses is valid for low levels of wall loss relative to the number of remaining ions, $n_{-1}$.

If diffusional loss or electric field capture of ions is significant, that is $\hat{B}_{v} \gg 0$ or $\hat{C}_{v} \gg 0$, a numerical model is recommended. If $\hat{B}_{v} \approx 1$ or $\hat{C}_{v} \approx 1$ or higher, ion capture is of the same order as the remaining ion flux, therefore the present linear approximation breaks down and a numerical or CFD model must be implemented. To determine if wall loss has a significant effect on the particle charge level, Eq. 17 may be solved with and without the effect of wall loss. For example, the ratio of mean particle charge with wall losses relative to recombination, $\hat{B}_{r}$, is defined as follows:

$$
\hat{B}_{r}=\frac{1+\sqrt{\hat{A}_{v}}}{1+\sqrt{\frac{\hat{A}_{v}}{1+\hat{B}_{v}}}}=\frac{\bar{q}(\text { recomb }+ \text { wall loss })}{\bar{q}(\text { recomb })}
$$

where the total residence time is function of flow rate and geometry. Analogous parameters are summarized in Table III for the effect electric field loss and photoionization on the mean particle charge level, $\bar{q}$. For $\hat{A}_{r} \approx 1$, recombination may be neglected, for $\hat{B}_{r} \approx 1$, wall loss may be neglected, and for $\hat{C}_{r} \approx 1$, electric field losses may be neglected. The effect of recombination, diffusional wall loss, and electric field capture relative to photoionization can be estimated by the ratio of $\hat{B}_{r}$ or $\hat{C}_{r}$ to $\hat{A}_{r}$.

A comparison of the the analytical model described above with the solution of the numerical, 0D equations is shown in Figure 4. The average steady state charge per particle in Eq. 17 decreases with increasing concentration owing to an increase in recombination. The 0D numerical model follows the work by Maisels et $a l^{22,23}$ and from Appendix B, and does not require the assumption of large charges for the mean charge approximation, instead solving for the concentration of each class of charge $q$. The analytical model described in section II B begins to deviate from the 0D numerical model when recombination is high enough to reduce average charge per particle to +1 average charger per particle. However, for charge levels above +1 average charge per particle, the analytical and numerical models agree. Recombination must be included at concentrations above around $N=1 \times 10^{2}$ $\mathrm{cm}^{-3}$. Figure 4 also shows an increase in steady state charges per particle with logarithmically increasing electric field strength $(0.008<E<0.8 \mathrm{~V} / \mathrm{cm})$ according to Eq. 17. While Fig. 4 shows the increasing effect of electric field on $q$, the assumptions in the analytical solution for electric field are no longer valid at electric field greater than $E \approx 0.15 \mathrm{~V} / \mathrm{cm}$ which corresponds with $\hat{C}_{v}=0.75$ in this case. 
TABLE II. Analytical estimates of ion and particle number flow rate and electrical current

\begin{tabular}{|c|c|c|c|c|}
\hline & \multicolumn{2}{|r|}{ Ion } & \multicolumn{2}{|c|}{ Particle } \\
\hline & Number Rate $\left[\frac{\#}{s}\right]$ & Electric Current $[\mathrm{A}]$ & Number Rate $\left[\frac{\#}{s}\right]$ & Electric Current $[\mathrm{A}$ \\
\hline $\begin{array}{l}\text { Outlet } \\
\text { Diffusion } \\
\text { Electric field }\end{array}$ & $\begin{array}{c}j_{\mathrm{o}, \mathrm{i}}=Q \bar{q} N \\
j_{\mathrm{d}, \mathrm{i}}=j_{\mathrm{o}, \mathrm{i}} \frac{\tau_{\mathrm{c}, \mathrm{i}}}{\tau_{\mathrm{d}, \mathrm{i}}} \\
j_{\mathrm{e}, \mathrm{i}}=j_{\mathrm{o}, \mathrm{i}} \frac{\tau_{\mathrm{c}, \mathrm{i}}}{\tau_{\mathrm{e}, \mathrm{i}}}\end{array}$ & $\begin{array}{c}i_{\mathrm{o}, \mathrm{i}}=j_{\mathrm{o}, \mathrm{i}} e \\
i_{\mathrm{d}, \mathrm{i}}=i_{\mathrm{o}, \mathrm{i}} \frac{\tau_{\mathrm{c}, \mathrm{i}}}{\tau_{\mathrm{d}, \mathrm{i}}} \\
i_{\mathrm{e}, \mathrm{i}}=i_{\mathrm{o}, \mathrm{i}} \frac{\tau_{\mathrm{c}, \mathrm{i}}}{\tau_{\mathrm{e}, \mathrm{i}}}\end{array}$ & $\begin{array}{c}j_{\mathrm{o}, \mathrm{p}}=Q N \\
j_{\mathrm{d}, \mathrm{p}}=j_{\mathrm{o}, \mathrm{p}} \frac{\tau_{\mathrm{c}, \mathrm{p}}}{\tau_{\mathrm{d}, \mathrm{p}}} \\
j_{\mathrm{e}, \mathrm{p}}=j_{\mathrm{o}, \mathrm{p}} \frac{\tau_{\mathrm{c}, \mathrm{p}}}{\tau_{\mathrm{e}, \mathrm{p}}}\end{array}$ & $\begin{array}{c}i_{\mathrm{o}, \mathrm{p}}=j_{\mathrm{o}, \mathrm{p}} \bar{q} e \\
i_{\mathrm{d}, \mathrm{p}}=i_{\mathrm{o}, \mathrm{p}} \frac{\tau_{\mathrm{c}, \mathrm{p}}}{\tau_{\mathrm{d}, \mathrm{p}}} \\
i_{\mathrm{e}, \mathrm{p}}=i_{\mathrm{o}, \mathrm{p}} \frac{\tau_{\mathrm{c}, \mathrm{p}}}{\tau_{\mathrm{e}, \mathrm{p}}}\end{array}$ \\
\hline
\end{tabular}

TABLE III. Dimensionless parameters

\begin{tabular}{|l|c|c|}
\hline & Parameter & $\begin{array}{c}\text { Effect on charge } \\
\text { state relative } \\
\text { to recombination }\end{array}$ \\
\hline Photocharging & $\hat{A}_{v}=\frac{4 h \nu D_{i} N}{K_{c} I V_{e} k_{\mathrm{B}} T}$ & $\hat{A}_{r}=1+\sqrt{\hat{A}_{v}}$ \\
$\hat{B}_{v}=\frac{\tau_{\mathrm{c}, \mathrm{i}}}{\tau_{d, i}}$ & $\hat{B}_{r}=\frac{1+\sqrt{\hat{A}_{v}}}{1+\sqrt{\frac{\hat{A}_{v}}{1+\hat{B}_{v}}}}$ \\
Diffusion & $\hat{C}_{v}=\frac{\tau_{\mathrm{c}, \mathrm{i}}}{\tau_{\mathrm{e}, \mathrm{i}}}$ & $\hat{C}_{r}=\frac{1+\sqrt{\hat{A}_{v}}}{1+\sqrt{\frac{\hat{A}_{v}}{1+\hat{C}_{v}}}}$ \\
\hline
\end{tabular}

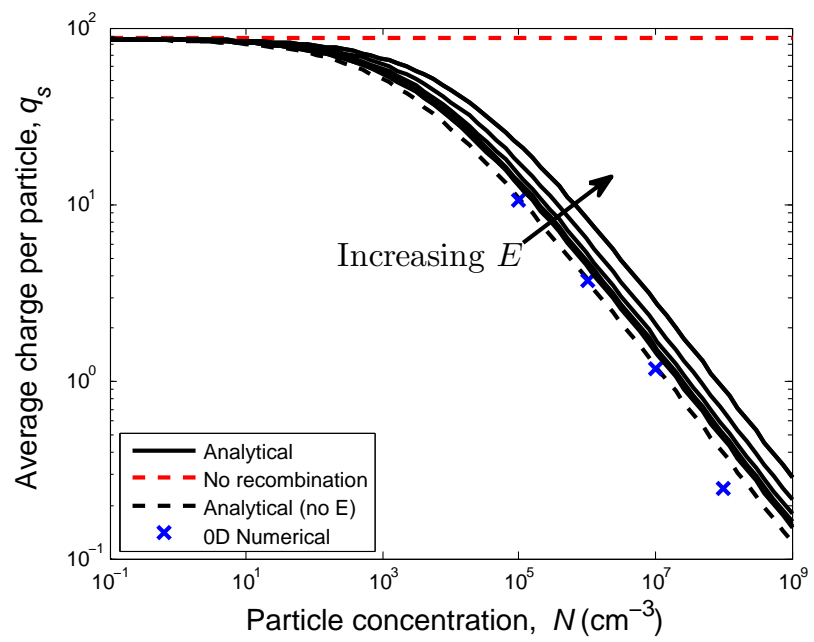

FIG. 4. Steady state charge for a monodisperse, singlecomponent aerosol as a function of concentration. Logarithmically increasing electric field strength $(0.008<E<0.8$ $\mathrm{V} / \mathrm{cm})$

\section{Limitations of Analytical Model}

The formulations of the analytical equations and dimensionless parameters assume a large, positive number of charges per particle and low ion wall losses relative to ion concentration. The main assumption within the charging equation (Eq. 7) is that the image charge has a negligible effect, which is true during the low charging regime $\bar{q} \gg 1$, otherwise the effect of image charge is significant and must be included. The main assumption in the recombination equation (Eq. 8) is that the charge level of the particle dominates the electrostatic attraction and the image force can be neglected, true for $\bar{q}>1$. In reducing the governing equations of Eq. 5 to the ion conservation in Eq. 9, large, postive charges per particle must be assumed. The assumptions that $n_{-1}$ is constant in the integration of Eq. 15 and that the ion losses are small in the formulation of Eq. 17 are no longer valid at high levels of wall loss relative to the remaining ions.

Although the analytical model is useful in thinking through the processes involved, and in quick calculations for sizing and field intensity for particle charging, numerical models are necessary to solve the conservation equations whenever the assumptions used in its derivation are violated, and losses are no longer linear.

\section{3D Computational Fluid Dynamics Model}

Equations for UV photoionization and ion/particle recombination are coupled with ion/particle advection and diffusion and electric field transport in three-dimensional CFD for the first time. Upwards of fifty simultaneous species transport equations can be solved if necessary to allow the resolution of local charge distribution and average charges per particle for multiple charge states. Results from the CFD model are verified using data from existing literature ${ }^{29}$ and a $0 \mathrm{D}$ numerical model described in Maisels et al. ${ }^{22,23}$ and Appendix B. Verification results are shown in Appendix B.

\section{D CFD Equations}

The velocity and pressure fields are solved in the form of the steady-state Navier Stokes equations in three dimensions. In this case, the carrier gas is considered to be air at NTP. The charged particles and ions are assumed not to affect the conservation equations of the carrier gas, that is, constant air density and viscosity, and negligible electro-hydrodynamic effects. The calculated flow field is imposed on the local particles and ions. The steady conservation equations for particle and ion concentrations is solved, as shown in Eqns. 5 and 6 for negatively charged ions and all particle charge levels, $q$, between $q_{\min }$ and 
$q_{\max }$. The iteration of the concentration equations is enabled by the objected-oriented nature of $\mathrm{C}++$ and user access to the source code and solvers of the CFD package used, OpenFOAM. The minimum charge level, $q_{\min }$, is set as -5 charges per particle, which is sufficiently low such that the concentrations of all neutral and negatively charged particles in this system are calculated. The maximum possible charge level from Eq. 3. is calculated at runtime to determine $q_{\max }$ from Eq. $6 \mathrm{~b}$ and the necessary number of simultaneous, coupled concentration transport equations is solved for each charge level, an approach which has not previously been reported.

A Laplacian equation is applied to calculate the potential field based on the voltage, $V$, at the boundaries. The low intensity electric field, $\vec{E}$, is calculated as the gradient of the potential field, as follows, and applied to Eq. 5.

$$
\begin{aligned}
\nabla^{2}\left(\varepsilon_{0} V\right) & =0 \\
\vec{E} & =\nabla \cdot V
\end{aligned}
$$

\section{Geometry and Mesh}

The geometry under consideration for a particle charger and detector is a $20 \mathrm{~cm}$ long cylinder of $25 \mathrm{~mm}$ diameter. The outer cylinder makes up one electrode and the second is a concentric rod of $1.5 \mathrm{~mm}$ in diameter and the full length of the cylinder. The computational mesh consists of a $1 / 8^{\text {th }}$ of a cylinder due to the axisymmetric nature of the solution. Figure 5 shows a distribution of charges per particle and illustrates the geometry of the case under consideration. The flow proceeds from the circular inlet to the outlet, along the axial direction. The photoionization process is assumed to take place as a uniform volumetric source throughout the cylinder, increasing the charges per particle. The assumption of uniform light intensity is an idealisation of a chamber without geometric effects or attenuation, such as by application of collimated light with negligible volumetric absorption. The light intensity properties can be tailored to specific geometric configurations or operating conditions. An electric field is applied which transports charged particles towards the centre rod, thereby increasing the concentration of highly charged particles near the rod. The bias voltage may be reversed to drive particles towards the outer cylinder and attract ions towards the center rod. The geometry under consideration yields $2 \mathrm{D}$ axisymmetric results. Therefore, although the governing equations are solved in 3D, they could be optimized for an axisymmetric solution. However, by solving in 3D, the model may be readily adapted to geometries with $3 \mathrm{D}$ effects.

The governing equations are converted to steady-state linear algebraic equations using OpenFOAM v.4. Based on a test of grid independence, the computational mesh consists of 230,400 cells which are graded near walls

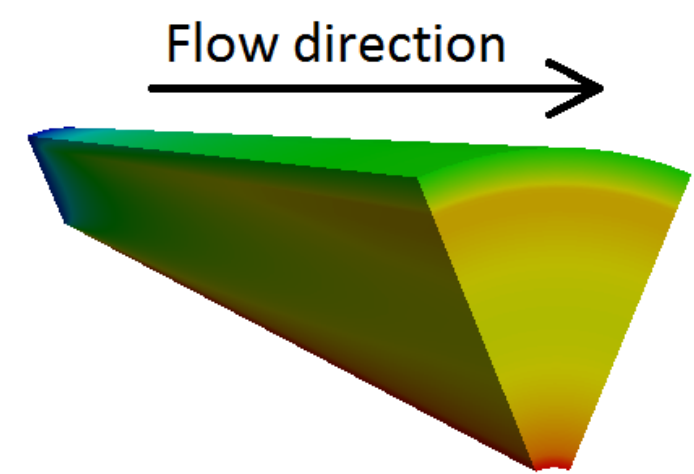

\section{Charges Per Particle}

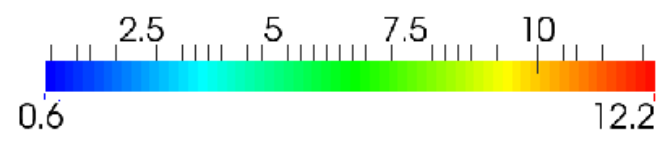

FIG. 5. Sample distribution of charges per particle during convective flow under irradiation. The charge level increases in the axial flow direction due to photoionization.

where the highest concentration gradients occur. The solution time depends on the number of coupled transport equations and charge level per particle. For a maximum charge of 14 charges per particle, a solution is computed in 11.5 minutes on a single core of an 8 core processor (Intel@) Core ${ }^{\mathrm{TM}}$ i7 $3.40 \mathrm{GHz}$ ) with $16 \mathrm{~GB}$ of RAM running on openSUSE 13.1. The computational mesh is not yet optimized and parallel processing can readily be incorporated if necessary.

\section{Boundary and Operating Conditions}

Initially neutral, monodisperse particles are entrained in a flow of air at NTP. A uniform velocity profile and fixed, neutral nanoparticle concentration is selected at runtime and prescribed at the inlet. Boundary conditions of zero gradient are applied for velocity and concentration of air at the outlet and set to zero (or no-slip) at the walls. The boundary conditions for momentum, particle, ion and carrier concentration and voltage equations are summarized in A1.

The effect of UV light is represented by intensity, $I$ (coupled with $K_{c}$ ), light energy, $h \nu$, particle work function, $\Phi_{\infty}$, and particle size as defined by Eqns. 1 and 2 applied as uniformly distributed source terms. The radiation is modeled as from a $185 \mathrm{~nm}(6.69 \mathrm{eV})$ wavelength source, greater than the work function of most solids. The particle diffusion coefficient is calculated as a function of particle diameter using the mechanical mobility and Cunningham slip correction factor as outlined in Ref. ${ }^{35}$ for air at NTP. The particle electrical mobility is a linear function of the diffusion coefficients for a given temperature ${ }^{35}$. A uniform ion mobility distribution 
is assumed as using properties gathered from Wiedensohler et al. ${ }^{36}\left(1.6 \times 10^{-4} \mathrm{~m}^{2} \mathrm{Vs}^{-1}\right)$ which is within the range of more recent ion mobility distribution measurements from Maißer et al. ${ }^{28}\left(1.5-1.9 \times 10^{-4} \mathrm{~m}^{2} \mathrm{Vs}^{-1}\right)$ and Steiner \& Reischl ${ }^{37}\left(0.9-2.5 \times 10^{-4} \mathrm{~m}^{2} \mathrm{Vs}^{-1}\right)$. The model parameters and operating conditions are summarized in Table IV.

TABLE IV. Model parameters and operating conditions

\begin{tabular}{|l|l|}
\hline Ion diffusivity $^{36}, D_{i}$ & $4.04 \times 10^{-6} \mathrm{~m}^{2} / \mathrm{s}$ \\
Ion electrical mobility ${ }^{36}, Z_{-1}$ & $1.6 \times 10^{-4} \mathrm{~m}^{2} \mathrm{Vs}^{-1}$ \\
Mean particle diameter, $d_{p}$ & $20 \mathrm{~nm}$ \\
Particle concentration, $N$ & $10^{6} \mathrm{~cm}^{-3}$ \\
Flow rate, $Q$ & $1 \mathrm{std} \mathrm{L} / \mathrm{min}$ \\
Mean velocity, $U$ & $3.45 \times 10^{-2} \mathrm{~m} / \mathrm{s}$ \\
Convection time, $\tau_{c}$ & $5.8 \mathrm{~s}$ \\
Empirical constant, $m$ & 2 \\
Photoemission constant, $K_{c} I$ & $1.9 \times 10^{35} \mathrm{~J}^{-1} \mathrm{~m}^{-2} \mathrm{~s}^{-1}$ \\
Light energy, $h v$ & $6.69 \mathrm{eV}(185 \mathrm{~nm})$ \\
Work function, $\Phi_{\infty}$ & $4.95 \mathrm{eV}$ \\
\hline
\end{tabular}

\section{DISCUSSION}

Equations for photocharging, recombination and transport of ultrafine particles are solved for continuous flow through a cylindrical chamber using 0D numerical and CFD models. For the conditions outlined in Table IV, with no electric field, the particles are clearly saturated with charge as $\tau_{c}=5.8 \mathrm{~s}>\tau_{s}=1 \mathrm{~s}$, and recombination is a dominant flux, as $\hat{A}_{r}=10.9$. Diffusional ion wall loss is significant, as $\hat{B}_{r}=1.24$ at zero bias voltage. Above around $0.25 \mathrm{~V}(0.2 \mathrm{~V} / \mathrm{cm})$, the electric field capture of ions is of the same order as the remaining ions, since at that voltage, $\hat{C}_{v}=0.9$. This regime requires a numerical or CFD model to quantify the effects of ion capture on particle charging.

Figure 6 shows a 2D slice of the computational domain where particles are flowing in the positive $\mathrm{z}$-direction and a voltage of $1 \mathrm{~V}(0.8 \mathrm{~V} / \mathrm{cm})$ is applied between the top and bottom electrodes. The concentration of ions of -1 charge and total particle concentration are shown for an inlet concentration of $10^{6} \mathrm{~cm}^{-3}$ neutral particles of $20 \mathrm{~nm}$ diameter. The ions generated during the photocharging process are transported and captured at the top, positive electrode and the charged particles are transported towards the bottom, negative electrode. Each remaining $2 \mathrm{D}$ slice shows the concentration of particles at a given mean charge, $q$, from 0 to 6 charges per particle. Particles are charged to higher charge levels as the flow proceeds in the z-direction while the particles are simultaneously transported to the bottom, negative electrode. The particles near the inlet are held at lower charge levels at 1 or 2 charges per particle due to the presence of ions in those areas.

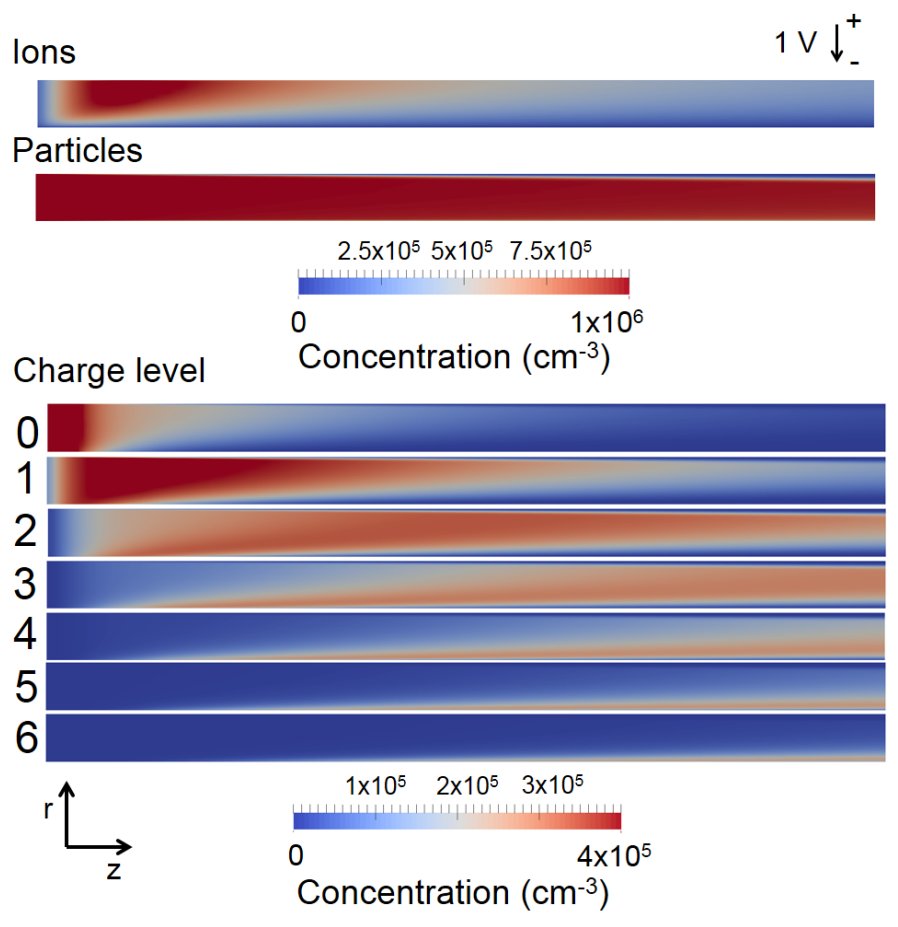

FIG. 6. (Top two panels) Steady-state concentration of total ions, $n_{-1}$, generated from photoionization of particles, $N$, across half-symmetry plane for the domain. (Bottom seven panels) Corresponding number concentration of particles with charge levels from 0 to 6 . Operating conditions are: inlet velocity of $0.0345 \mathrm{~m} / \mathrm{s}$ in the +z direction, particle inlet concentration of $N=10^{6} \mathrm{~cm}^{-3}$, neutral particles with a monodisperse diameter of $20 \mathrm{~nm}$. A voltage of $1 \mathrm{~V}$ is applied between the top (cylinder) and bottom (rod) electrode.

A positive bias voltage indicates that the outer cylinder has a positive potential relative to the rod, and the reverse is true for a negative bias voltage. For a range of bias voltages, the total current, $i_{\mathrm{e}}$, is calculated from the total (diffusive) flux of ions and particles integrated at the interface of the rod and outer cylinder walls. The outlet current, $i_{\mathrm{o}}$, is also calculated from the total (diffusive plus convective) flux of positively plus negatively charged ions passing through the outlet, multiplied by their respective charges.

The results for the 0D numerical model and 3D CFD model with positive and negative biases are shown in Fig. 7. At bias voltages around $1 \mathrm{~V}(0.8 \mathrm{~V} / \mathrm{cm})$, only the highly mobile ions are transported to either the rod or cylinder leaving less opportunity to recombine with the charged particles, and resulting in higher currents for both $i_{\mathrm{e}}$ and $i_{\mathrm{o}}$. Every positive charge on a particle creates an equal and opposite charge on a negative ion, therefore at voltages around $1 \mathrm{~V}(0.8 \mathrm{~V} / \mathrm{cm}), i_{\mathrm{e}}$ and $i_{\mathrm{o}}$ are of equal magnitude. As the bias voltage increases above approximately $2.5 \mathrm{~V}(2 \mathrm{~V} / \mathrm{cm})$, the flux of charged particles near the rod or outer cylinder begins to dominate, thereby decreasing the outlet current, $i_{\mathrm{O}}$. The current, $i_{\mathrm{e}}$, continues to increase as the negatively charged ions 
are collected at the rod and positively charged particles are collected at the outer cylinder, both contributing to current $i_{\mathrm{e}}$ as shown in Fig. 8. The numerical model does not capture the differences between a positive and negative bias. At low, negative bias voltage, below around $1 \mathrm{~V}(0.8 \mathrm{~V} / \mathrm{cm})$, ions preferentially diffuse to the outer cylinder rather than the centre rod due to the difference in electrode surface area creating an effective current. At low bias voltage, ion diffusion is faster than the electric field flux. At $0.05 \mathrm{~V}(0.04 \mathrm{~V} / \mathrm{cm})$, the calculated ratio of characteristic times in Eq. 14, $\hat{\tau}_{e d, i}=0.5$, whereas at 1 $\mathrm{V} \hat{\tau}_{e d, i}=10$ and the electric field flux dominates.

The average charge per particle and charge distribution at the outlet are shown in Fig. 9 and Fig. 10, respectively, as a function of bias voltage. Particle charge levels can be controlled by changing the bias voltage, thus inducing more or less direct removal of ions from the control volume, resulting in correspondingly different opportunity for recombination. At high voltages, charged particles as well as ions can be removed, thereby changing the charge distribution and mean charge at the outlet. In these cases, the mean charges can be controlled between 2 and 7 charges per particle. At low bias voltages, below around $1 \mathrm{~V}(0.8 \mathrm{~V} / \mathrm{cm})$, the calculated local charge per particle using the $3 \mathrm{D}$ CFD model increases significantly nearest the walls where the highest diffusion loss of ions occurs as shown in Figs. 10a and b. As a result, the rate term of the $0 \mathrm{D}$ numerical model does not adequately capture the effect of diffusional ion loss on average charge per particle at low bias voltages. At bias voltages from $1 \mathrm{~V}(0.8 \mathrm{~V} / \mathrm{cm})$ to $10 \mathrm{~V}(8 \mathrm{~V} / \mathrm{cm})$, ions are captured quickly, particles reach higher charge states and are transported towards the the negative electrodes as seen in Figs. 10b and c. At high bias voltages, above around $10 \mathrm{~V}(8 \mathrm{~V} / \mathrm{cm})$, highly charged particles localized near the negative electrode are captured more readily by the applied electric field as seen in Fig. 10d, leading to a lower average charge per particle at the outlet and higher current, $i_{e}$, relative to the $0 \mathrm{D}$ numerical model. The latter does not solve local charge levels, but rather a rate term for the electric field capture as described in Table B1 of Appendix B. Without a local resolution of charge levels, the 0D numerical model cannot adequately capture the current or particle charging at low and high bias voltage conditions.

\section{CONCLUSIONS}

The present work introduces the first analysis of simultaneous photoionization, recombination, advection/diffusion and electrical field transport of multiplycharged particles through a steady state control volume using analytical and numerical techniques. Analytical equations are used to define characteristic times for photoionization, recombination, convection, diffusion, and electric field transport mechanisms allowing the formulation of a range of dimensionless parameters to understand

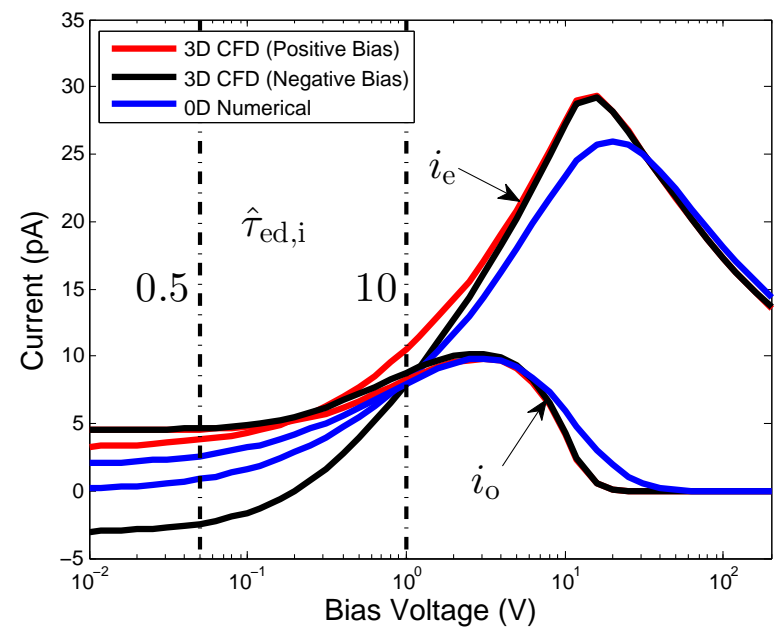

FIG. 7. Ion and particle current, $i_{\mathrm{e}}$, and outlet current, $i_{\mathrm{o}}$, as a function of applied bias voltage for numerical and CFD models. A positive bias indicates outer cylinder is at a positive voltage relative to rod, and the converse for negative bias.

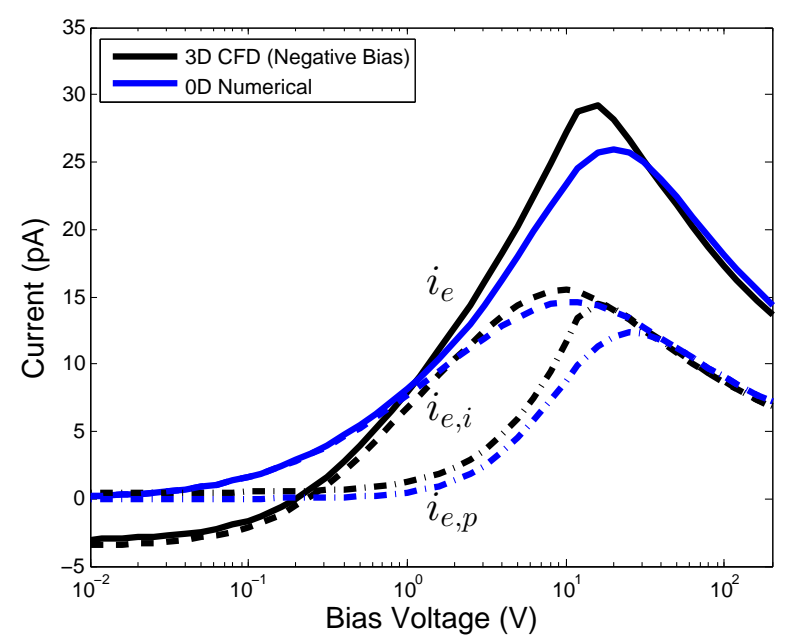

FIG. 8. Ion and particle current, $i_{\mathrm{e}}$, made up of the sum of ion current, $i_{\mathrm{e}, \mathrm{i}}$, and particle current $i_{\mathrm{e}, \mathrm{p}}$ as a function of applied bias voltage for numerical and CFD models.

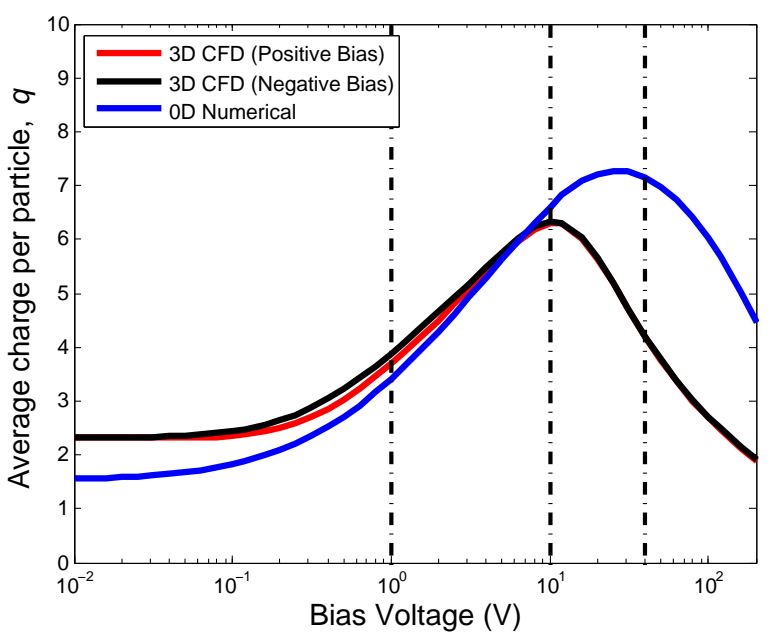

FIG. 9. Average charge per particle at outlet as a function of bias voltage for numerical and CFD models. The dotted lines mark the voltages at which the charge distributions are presented in Fig. 10. 
$\log$ of concentration $\left(\mathrm{cm}^{-3}\right)$

(a) OV

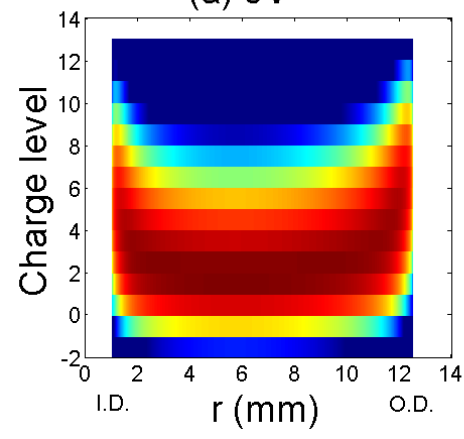

(b) $1 \mathrm{~V}$

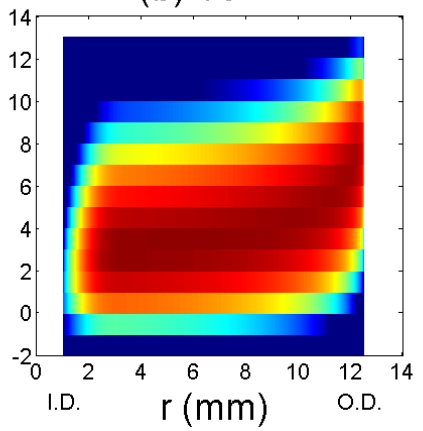

(c) $10 \mathrm{~V}$

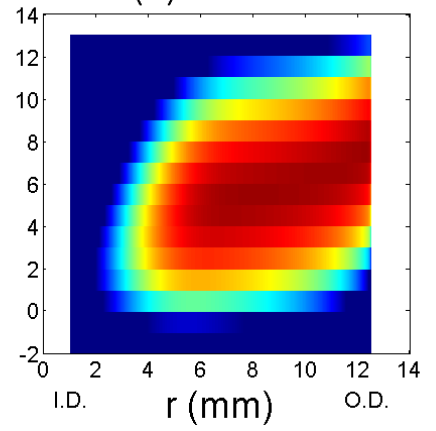

(d) $40 \mathrm{~V}$

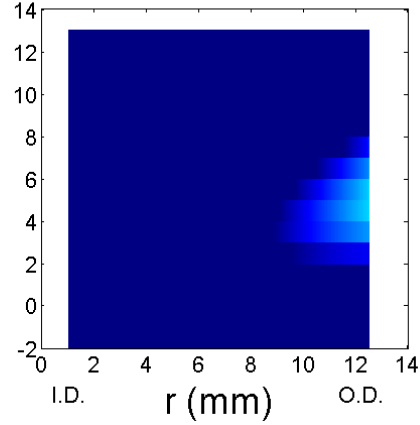

FIG. 10. Charge distribution of discrete particle charges at the chamber outlet as a function of radial distance from the centerline at four different voltages; (a) $0 \mathrm{~V}$, (b) $1 \mathrm{~V}$, (c) $10 \mathrm{~V}$, and (d) $40 \mathrm{~V}$ between the rod (I.D., positive electrode) and cylinder (O.D., negative electrode).

the relative contributions to the particle flux balance. Dimensionless parameters are defined to determine when the effects of electric field or diffusional ion or particle loss require a more computationally expensive numerical model or when the effects may be neglected. The assumptions for the analytical model are shown to be valid for a range of simple conditions of interest, as well as for system dimensioning. However, a more detailed numerical model including detailed charge accounting is required for average levels of charge per particle at or below +1 charges, as well as high ion losses. The computational fluid dynamics (CFD) model developed in this work is the first to include equations capturing UV photoionization and detailed ion and particle recombination theory. The $0 \mathrm{D}$ numerical and 3D CFD models agree well for a large range of electric field strengths for the simple geometry of concentric cylinders, but differences appear at both high (above $\approx 8 \mathrm{~V} / \mathrm{cm}$ ) and low (below $\approx 0.8 \mathrm{~V} / \mathrm{cm}$ ) electric field strengths, where the detailed spatial resolution rather than a simple linear 0D model becomes necessary. The 0D numerical and 3D CFD results both demonstrate that the average particle charge level, and the resulting charges at the electrodes and ends of the system can be controlled by changing the bias voltage. These features can be used in the development of a particle discrimination and measurement system, and will be explored in future papers.

\section{ACKNOWLEDGMENTS}

The authors gratefully acknowledge Alphasense Ltd. and Cambridge Trust for financial support.

\section{Appendix A: CFD Model Boundary Conditions}


TABLE A1. Boundary conditions for the conservation equations

\begin{tabular}{|l|l|l|l|}
\hline Type & Momentum & Concentration & Voltage \\
\hline Inlet & $u=U, \frac{\partial p}{\partial n}=0$ & $N_{0}=N_{0, i n}, N_{q \neq 0}=0, n_{i}=0$ & $\frac{\partial V}{\partial n}=0$ \\
\hline Outlet & $\frac{\partial u}{\partial n}=0, p=1.01325$ bar & $\frac{\partial N_{q}}{\partial n}=0, \frac{\partial n_{i}}{\partial n}=0$ & $\frac{\partial V}{\partial n}=0$ \\
\hline Rod & $u=0, \frac{\partial p}{\partial n}=0$ & $N_{q}=0, n_{i}=0$ & $V=V_{\mathrm{r}}$ \\
\hline Outer cylinder & $u=0, \frac{\partial p}{\partial n}=0$ & $N_{q}=0, n_{i}=0$ & $V=V_{h}$ \\
\hline Angular Symmetry & cyclic & cyclic & cyclic \\
\hline
\end{tabular}




\section{Appendix B: Model Verification}

The 0D numerical model is verified against existing numerical models from Hoppel and Frick ${ }^{38}$, and Maisels et $a l .{ }^{22}$. In the 0D numerical model, the divergence terms for advection, diffusion and electric field transport in Eq. 5 are replaced by rate terms as shown in Table B1.

TABLE B1. Terms in governing equations

\begin{tabular}{|l|l|l|}
\hline & $3 \mathrm{D}$ CFD & 0D numerical \\
\hline Particles & $\nabla \cdot\left(\vec{u} N_{q}\right)$ & $\frac{d N_{q}}{d t}$ \\
& $\nabla \cdot\left(D_{p} \nabla N_{q}\right)$ & $-\frac{4 D_{p}}{R^{2}} N_{q}$ \\
& $\nabla \cdot\left(Z_{q} \vec{E} N_{q}\right)$ & $-\frac{Z_{p} E}{R} N_{q}$ \\
\hline Ions & $\nabla \cdot\left(\vec{u} n_{j}\right)$ & $\frac{d n_{j}}{d t}$ \\
& $\nabla \cdot\left(D_{i} \nabla n_{j}\right)$ & $-\frac{4 D_{i}}{R^{2}} n_{j}$ \\
& $\nabla \cdot\left(Z_{j} \vec{E} n_{j}\right)$ & $-\frac{Z_{j} E}{R} n_{j}$ \\
\hline
\end{tabular}

\section{OD NUMERICAL MODEL}

The 0D numerical model is compared against a numerical model from Hoppel and Frick ${ }^{38}$ for the concentration of ions and charged particles over time and shown in Fig. A.1. There is a constant generation rate of positive $\left(n_{+}\right)$and negative ions $\left(n_{-}\right)$in a 0D case. The ions transfer charge to the particles through a diffusion charging process. The differences in results at the steady state values relate to the assumptions made in the collision kernels of Hoppel and Frick ${ }^{38}$. The 0D numerical model contains a more detailed calculation of mean free path (Reischl et al. 1996 ${ }^{34}$ ) and other properties.

An analytical equation from Wiedensohler ${ }^{39}$ estimates the Fuchs distribution found after the recombination process reaches an equilibrium. The fractions of multiply charged particles are calculated using the 0D numerical model with ion mobilities from Wiedensohler ${ }^{39}$. Fig. A.2 shows the 0D numerical model matches Fuchs equilibrium charge distribution after a sufficient charging time. The differences relate to the assumptions made in the analytical equation of Wiedensohler ${ }^{39}$.

Charge level per particle is shown as a function of time for a range of concentrations in Fig A.3. The aerosol particles are irradiated with UV light which causes an increase in charges per particle. At high concentrations, recombination is more likely, thereby decreasing the charging per particle. An equilibrium state is reached in which photoionization rate matches the recombination rate. The aerosol is no longer irradiated beyond $t=0.71 \mathrm{~s}$,

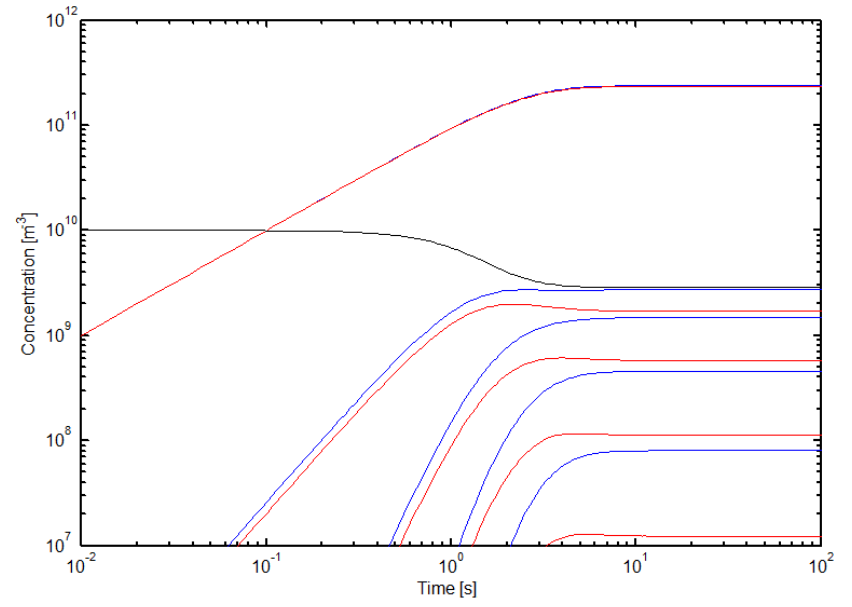

FIG. A.1. Asymmetric diffusion charging of particles $\left(\mathrm{D}_{p}=\right.$ $200 \mathrm{~nm}$ ) for ionization rate $10^{5}$ ion pairs $\mathrm{cm}^{-1} \mathrm{~s}^{-1}$.

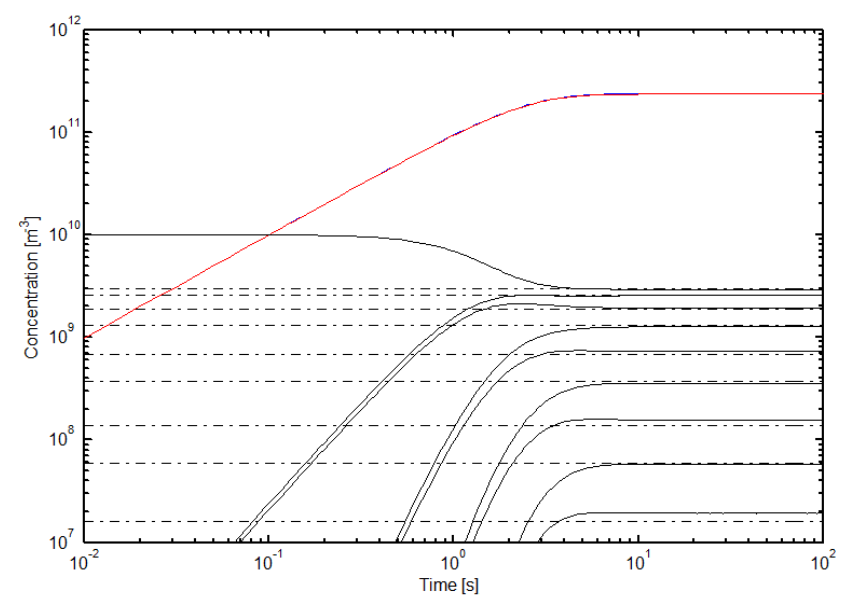

FIG. A.2. 0D numerical - Asymmetric diffusion charging of particles $\left(D_{p}=200 \mathrm{~nm}\right)$ for ionization rate $10^{5}$ ion pairs $\mathrm{cm}^{-1}$ $\mathrm{s}^{-1}$. Photoionization is turned off in this case. The fraction of multiply charged particles in a Fuchs distribution are estimated using an analytical equation from Wiedensohler, 1988 $\left(\right.$ dotted lines) ${ }^{39}$.

therefore recombination dominates. The present $0 \mathrm{D}$ numerical model shows excellent agreement with the numerical model of Maisels et al. ${ }^{22}$.

\section{COMPUTATIONAL FLUID DYNAMICS}

Equations for photoionization and recombination theory are applied in 3D CFD and verified against existing numerical models from Hoppel and Frick ${ }^{38}$ and the 0D numerical model. The equations were first applied in a 1-D CFD model in which boundary effects were neglected for comparison.

Results from the numerical model from Hoppel and 


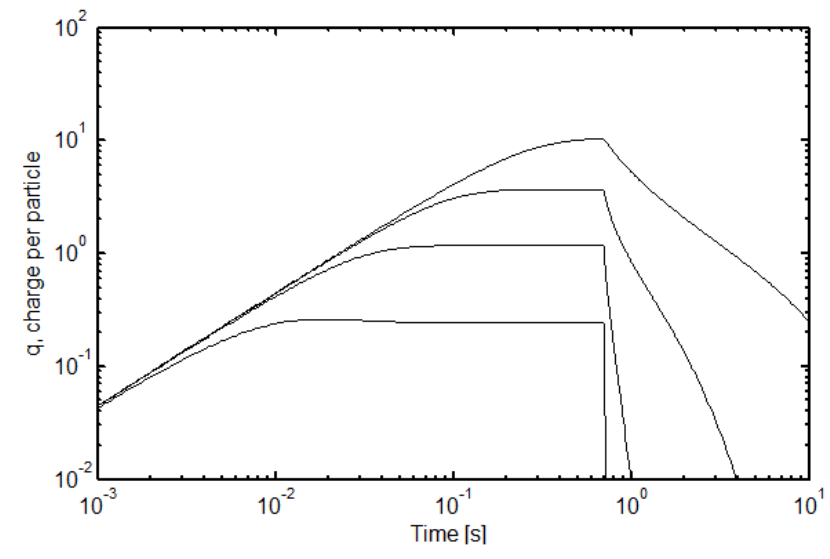

FIG. A.3. Photoionization is combined with the diffusion charging process. The $0 \mathrm{D}$ numerical model corresponds to the dots of Maisels et al. which represent the numerical model. The solid lines in et al. ${ }^{22}$ represent an analytical model which is only valid for high charging levels. The aerosol flow leaves the irradiated region at $\mathrm{t}=0.71 \mathrm{~s}$.

Frick $^{38}$ are compared against the CFD model for the concentration of ions and charged particles over time and shown in Figure A.4. There is a constant generation rate of positive $\left(\mathrm{n}_{+}\right)$and negative ions $\left(\mathrm{n}_{-}\right)$in a 1-D case. The ions transfer charge to the particles through a diffusion charging process. The charging is asymmetric due to the increased mobility of the negative ions over the positive ions. Photoionization is turned off in this case.

The 0D numerical and CFD models are compared for photoionization and recombination processes and results are shown in Figures A.5. Negative ions and positively charged particles are produced due to photoionization. Concentrations of particles at higher charge levels become more significant as photocharging proceeds until an equilibrium state is reached. The agreement between models is excellent.

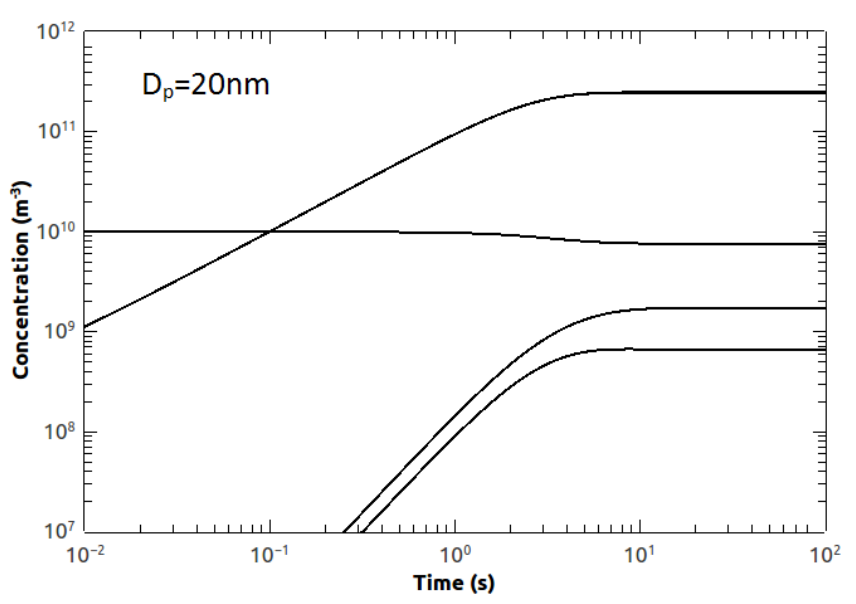

(a)1D CFD

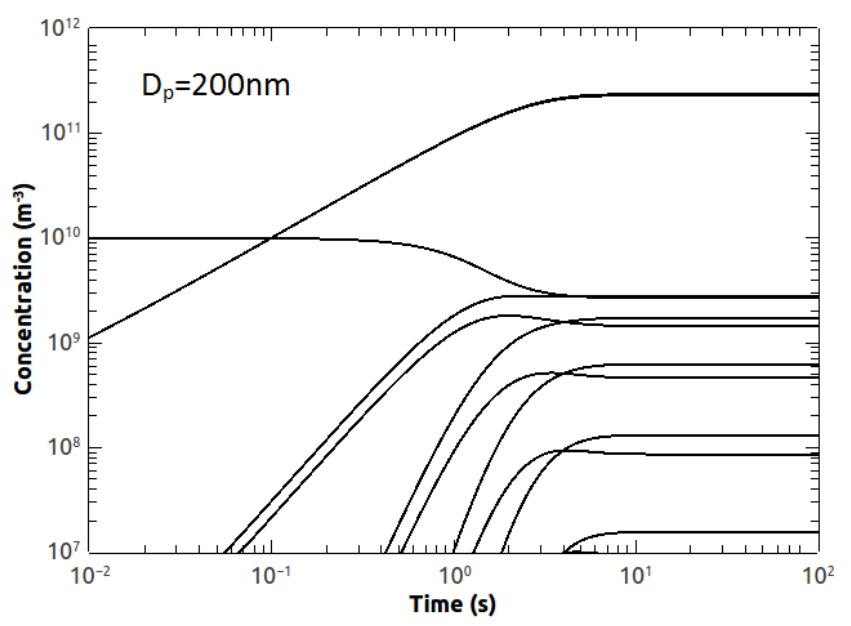

(b)1D CFD

FIG. A.4. Asymmetric diffusion charging of particles of diameter for ionization rate $10^{5}$ ion pairs $\mathrm{cm}^{-1} \mathrm{~s}^{-1}$. Photoionization is turned off in this case. Results match Hoppel and Frick $^{38}$. 


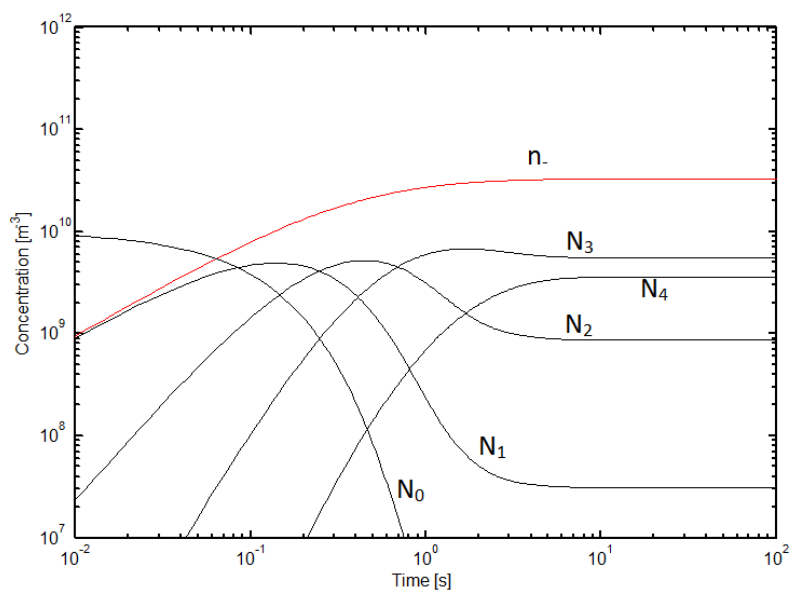

(a)0D numerical

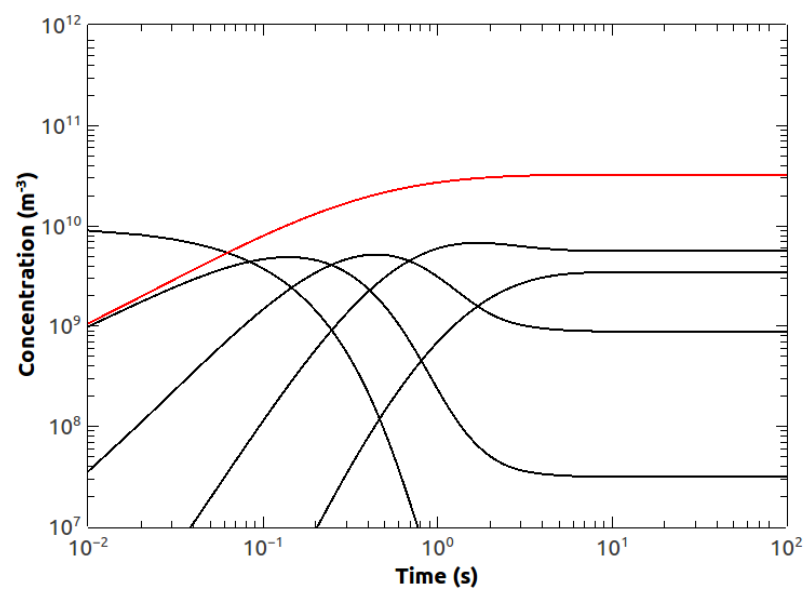

(b)1D CFD

FIG. A.5. Photoionization and recombination of particles $\left(\mathrm{D}_{p}\right.$ $=10 \mathrm{~nm}$ ). 
${ }^{1}$ A. Schmidt-Ott and H. C. Siegmann, Applied Physics Letters 32, 710 (1978).

${ }^{2} \mathrm{~T}$. Jung, H. Burtscher, and a. Schmidt-Ott, Journal of Aerosol Science 19, 485 (1988).

${ }^{3}$ M. Mohr, D. Matter, and H. Burtscher, Aerosol Science and Technology 24, 14 (1996).

${ }^{4}$ L. Li and D. R. Chen, Aerosol and Air Quality Research 11, 791 (2011).

${ }^{5}$ H. Burtscher, A. Schmidt-Ott, and H. C. Siegmann, Aerosol Science and Technology 8, 125 (1988).

${ }^{6}$ R. Niessner, Journal of Aerosol Science 17, 457 (1986).

${ }^{7}$ D. Matter, M. Mohr, W. Fendel, a. Schmidt-Ott, and H. Burtscher, Journal of Aerosol Science 26, 1101 (1995).

${ }^{8} \mathrm{~J}$. Jiang, M. H. Lee, and P. Biswas, Journal of Electrostatics 65 , 209 (2007).

${ }^{9}$ M. Cardona and L. Ley, Topics in Applied Physics 26 (1978).

${ }^{10}$ H. Burtscher, L. Scherrer, H. C. Siegmann, A. Schmidt-Ott, and B. Federer, Journal of Applied Physics 53, 3787 (1982).

${ }^{11}$ D. M. Wood, Physical Review Letters 46, 749 (1981).

${ }^{12}$ A. Schmidt-Ott and B. Federer, Surface Science Letters 106, A190 (1981).

${ }^{13}$ H. Burtscher, R. Niessner, and A. Schmidt-Ott, in Aerosols, Science, Technology and Industrial Applications of Airborne Particles (Elsevier New York, 1984) pp. pp. 443-446.

${ }^{14}$ H. Burtscher, R. Niessner, and A. Schmidt-Ott, in s: Science, Technology and Industrial Applications of Airborne Particles (Elsevier New York, 1984) pp. pp. 436-438.

${ }^{15}$ S. McDow, W. Giger, H. Burtscher, A. Schmidt Ott, and H. C. Siegmann, 24, 2911 (1990).

${ }^{16} \mathrm{M}$. Rosatzin and H. Burtscher, Forensic science international 120, 160 (2001).

${ }^{17}$ U. Matter, H. C. Siegmann, and H. Burtscher, Environmental Science \& Technology 33, 1946 (1999).

${ }^{18}$ U. Müller, A. Schmidt-Ott, and H. Burtscher, Zeitschrift für Physik B Condensed Matter 73, 103 (1988).

${ }^{19}$ U. Müller, H. Burtscher, and A. Schmidt-Ott, Physical Review B 38, 7814 (1988).

${ }^{20}$ B. Schleicher, H. Burtscher, and H. C. Siegmann, Applied
Physics Letters 63, 1191 (1993).

${ }^{21}$ E. Hontañón and F. E. Kruis, Aerosol Science and Technology 42, 310 (2008).

${ }^{22}$ A. Maisels, F. Jordan, and H. Fissan, Journal of Applied Physics 91, 3377 (2002).

${ }^{23}$ A. Maisels, F. Jordan, and H. Fissan, Journal of Aerosol Science 34, 117 (2003).

${ }^{24}$ T. Van der Zwaag, S. Haep, and K. G. Schmidt, Powder Technology 180, 97 (2008).

${ }^{25}$ J. Jiang, C. J. Hogan, D. R. Chen, and P. Biswas, Journal of Applied Physics 102 (2007), 10.1063/1.2768061.

${ }^{26}$ A. Leonardi, Feinste Schwebeteilchen aus Dieselmotoren, Ph.D. thesis (1991)

${ }^{27}$ L. Zhou and M. R. Zachariah, Chemical Physics Letters 525526, 77 (2012).

${ }^{28}$ A. Maißer, J. M. Thomas, C. Larriba-andaluz, S. He, and C. J. Hogan, Journal of Aerosol Science 90, 36 (2015).

${ }^{29}$ W. A. Hoppel and G. M. Frick, Aerosol Science and Technology 5, 1 (1986).

${ }^{30}$ N. A. Fuchs, Geofisica Pura e Applicata 56, 185 (1963).

${ }^{31}$ Y. G. Stommel and U. Riebel, $\mathbf{6 8 2 6}$ (2007), $10.1080 / 02786820701501873$.

${ }^{32}$ X. López-Yglesias and R. C. Flagan, Aerosol Science and Technology 47,688 (2013).

${ }^{33}$ R. Gopalakrishnan, M. J. Meredith, C. Larriba-Andaluz, and C. J. H. Jr., Journal of Aerosol Science 63, 126 (2013).

${ }^{34}$ G. P. Reischl, J. M. Mäkelä, R. Karch, and J. Necid, Journal of Aerosol Science 27, 931 (1996).

${ }^{35}$ P. Kulkarni, P. Baron, and K. Willeke, Wiley (2011).

${ }^{36}$ A. Wiedensohler, E. Lütkemeier, M. Feldpausch, and C. Helsper, Journal of Aerosol Science 17, 413 (1986).

${ }^{37}$ G. Steiner, T. Jokinen, H. Junninen, M. Sipila, T. Petaja, D. Worsnop, G. P. Reischl, and M. Kulmala, Aerosol Science and Technology 48, 261 (2014).

${ }^{38}$ W. A. Hoppel and G. M. Frick, Aerosol Science and Technology 12, 471 (1990)

${ }^{39}$ A. Wiedensohler, Journal of Aerosol Science 19, 387 (1988). 